\title{
A Fuzzy Logic Model to Evaluate The LeaN LEVEL OF AN ORGANIZATION
}

\author{
A. Abreu ${ }^{1}$ and J. M. F. Calado ${ }^{2}$ \\ ${ }^{1,2}$ Mechanical EngineeringDepartment, ISEL - Instituto Superior de Engenharia de \\ Lisboa, IPL - PolytechnicInstituteofLisbon Rua Conselheiro Emídio Navarro, 1, 1959- \\ 007 Lisboa, Portugal \\ ${ }^{1}$ CTS - Uninova - Instituto de Desenvolvimento de Novas Tecnologias \\ ${ }^{2}$ IDMEC/LAETA, Instituto Superior Técnico - Universidade de Lisboa
}

\begin{abstract}
This paper is concerned with the development of a holistic fuzzy logic model to evaluate the lean thinking environment in an organization. The development of such a model was based on a qualitative assessment approach, including quantitative basis, whose development has been based on fuzzy logic reasoning. Recourse to the use of fuzzy logic is justified by its ability to cope with uncertainty and imprecision on the input data, as well as, could be applied to the analysis of qualitative variables of a system, turning them into quantitative values. The proposed approach was structured with the aim of to achieve a model able to cope with the specificities of any kind of organization regardless of their nature, size, strategy and market positioning. Furthermore, the proposed methodology allows the systematically identification of constraint factors existing in an organization and, thus, provide the necessary information to the manager to develop a holistic plan for continuous improvement. To evaluate the robustness of the proposed approach, the methodology was applied to a maintenance and manufacturing aeronautical organization.
\end{abstract}

\section{KEYWORDS}

Data-driven models; Quantitative models; Qualitative models; Fuzzy logic; Lean thinking; Business analytics.

\section{INTRODUCTION}

All organizations are aiming for success. Today, success is measured by revenue growth, leadership in the market share supported by customer satisfaction and innovation capacity $[1,2]$. The increase in the globalization of markets combined with the new industrial revolution known as Industry 4.0 has led to profound changes in the way organizations operate [3]. The European framework program HORIZON 2020 stresses the need for organizations in the European area to equip themselves with management tools to accelerate productivity and innovation [4].The markets are increasingly globalized and competitive, constantly changing and evolving. Tight competition reduces opportunities for success. Social networks and the media emphasize success and expose the failures of organizations.

According to some studies, on average $40 \%$ of the costs in any organization is pure fat. The average efficiency of administrative procedures is less than 1\%. Eliminate this fat enables organizations to not only reduce costs and have more flexible and agile processes [5]. Lean Management is concerned with improving flows by seeking to reduce and/or eliminate activities that do not add value called "fats"[6]. 
In this context, the Lean Management is considered the excellent management tool to tackle the challenges of the twenty-first century whose motto is do more with fewer resources. To this end, many tools have been developed to support the implementation of a lean environment in organizations, which have been tested and used in industry [7] and in the services [6,8,9]. These tools have contributed to the simplification of procedures and created conditions for the reduction or elimination of waste ("fat") throughout the organization and create value for all stakeholders.

However, according to Bashin and Burcher [10], more than $90 \%$ of companies that have been applying lean tools and methodologies show shortcomings in the evaluation of their improved performance. The causes cited for this gap in the evaluation of performance improvements resulting from the lean approach implementation, are largely due to a lack of understanding of the concept of lean performance and appropriate models to monitor, evaluate and compare the evolution of "lean level" during the corresponding implementation process $[11,12,13]$.The lack of a clear understanding about what is lean performance and its evaluation is one of the reasons for lean programs implementation have failed. In other words, it is not possible to manage the lean level of an organization without measuring its performance.

Thus, as an instrument to support a high-performance management, it is urgent to develop a tool that allows measuring the organizational "fat" and that shows the level of waste according to a holistic perspective.

The measurement is the first step leading to the control and eventually to improvement of processes. If you do not measure, you do not understand. If you do not understand it, you cannot control it and if you cannot control it, you cannot improve it. The development of tools for assessing the leanness, or lack of fat, of an organization, are thus essential elements for the successful implementation of a lean culture. $[13,14,15]$ Therefore, one of the great challenges that this area faces is related to the development of models that allow to evaluate and validate the effectiveness and efficiency of the implementation of lean thinking in organizations in a holistic way with the possibility of simultaneously performing a qualitative and quantitative analysis.

The more rigorous and comprehensive be the developed model, the more likely it is to build valid and useful instruments, as in other areas of knowledge. For example, in the field of mechanical engineering, when the designer intends to design a new vehicle, he uses consolidated models that allow him to not only dimension the entire structure in order to meet the functional requirements, but also simulate the behaviour of that vehicle under conditions very close to the real ones. So one of the big challenges that this knowledge domain is facing, it is related to the need to build a holistic model for the assessment of organizational fat level. Only by having an organizational fat assessment model capable of measuring and aggregating the various fats in an organization according to several perspectives and simple to use and maintain, it is possible to detect and characterize in advance those aspects that the management should be aware, provide guidance for carrying out improvement actions and detect behaviours that can compromise the transformation of a fat organization in a lean organization, which means in a good "physical condition" to successfully face adversity in the world today.

In this context, this paper presents a model based on fuzzy logic that aims to determine the lean level of an organization, facing the challenges mentioned above and trying to overcome the corresponding difficulties, which could be seen as a modelling and decision-making tool for complex systems.

Hence, the paper is structured as follows:On section 2 is discussed the need to measure the lean level of an organization and also main distinct perspectives to assessing the level of leanness that has been suggested in literature. Section 3 presents a detailed description of the lean assessment approach proposed in this paper; to assess the robustness of the proposed method, on Section 4 is 
described its application to a case study and a critical analysis of the achieved results is provided; Section 5 includes some concluding remarks.

\section{SOME BACKGROUND}

Nowadays, when the subject of productivity and business success is addressed, there are several relevant issues that are the subject of research, for example: Why are not all organizations successful? Why the success formulas do not apply equally in all situations? What does it mean to be lean? How lean are the processes of our organization? How can my organization quantify the fats identified? And what is the most appropriate "fat" evaluation model in order to monitor strategies for increasing productivity and continuous improvement, which means implementing an anti-fat treatment in an organization? $[5,12,16]$

The "fat" in an organization is stored in its processes, propagates through its culture and originates in the attitudes and behaviours of its employees, since it is the collaborators who create the culture and develop the processes that store the fat [17].

According to some authors [18] e [19] can identify three distinct types of economic environment in relation to the level of competitiveness between organizations: markets "blue ocean", "red ocean and purple ocean An analogy is established between the phase diagram that is used in the thermodynamics field (showing the conditions necessary for the existence of the various states: solid, liquid and gaseous as a function of the pressure and temperature conditions) and the possible admissible levels of organizational fat associated with each market to not jeopardize the survival of an organization (obese, pre-obese and lean depending on the value of the opportunities and threats), as illustrated in Figure 1.
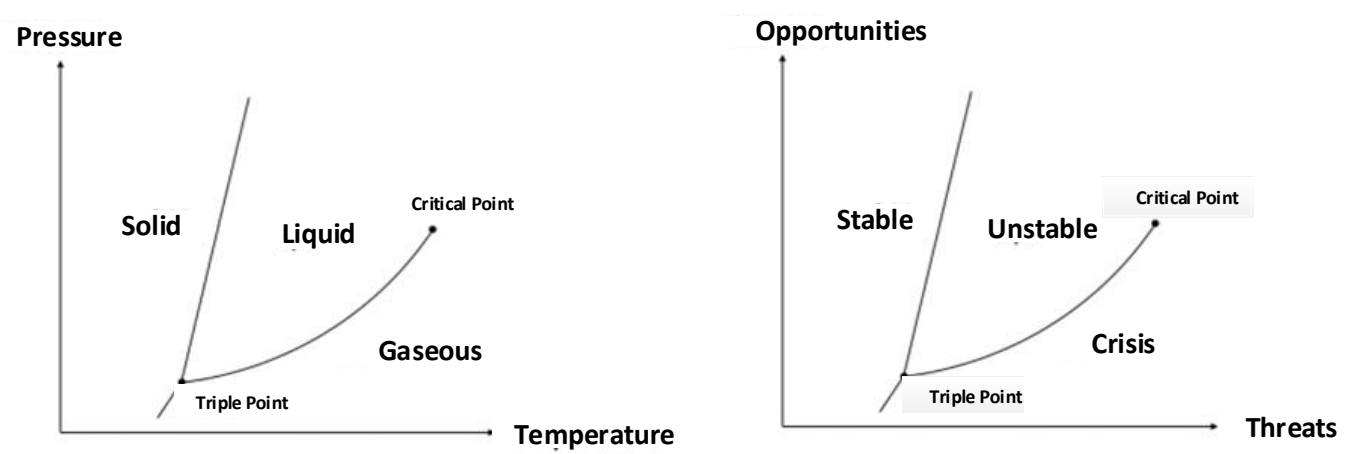

Figure 1. Diagram of Phases vs. Diagram of Market States.

The markets called "blue ocean", stable environment, correspond to the solid phase where the number of competitors is reduced and in some situations, could be null. In this favourable context, which is characterized by the ease of accessing many opportunities in contrast to the reduced number of threats, it provides the conditions for an organization to gain weight. However, the accumulation of fat that tends to increase over time does not significantly affect the performance of the organization to the point of immediately placing it in a crisis. In this context, the organization feels successful. The level of organizational fatness is not a concern of top management which leads to the proliferation of ineffective processes and the spread of a culture of self-indulgence. 
As opposed to the "blue ocean" market we have the markets called "red ocean", context of crisis, which corresponds to the "gaseous" state where the level of competition is extremely high. In this unfavourable context, the opportunities are scarce and the threats abound. Organizations try to outgrow their rivals in order to gain more and more market share.

Top management has as its main concern implementing strategies that ensure high performance to ensure the difficult and painful survival in these red oceans resulting from blood spilled in the competitive and death-generating fights for the less prepared organizations. Only one organization in shape, which means lean, has the artefacts needed to win the competition for the few opportunities that arise, while some of its competitors disappear.

In this adverse context, obese organizations feel frightened. The obese organizations either faces an accelerated weight-loss regime or faces death. To survive, they must change their processes, their culture and the attitudes and behaviour of their employees.

As an intermediate state, between the "blue ocean" market and the "red ocean" market there are the markets "purple ocean", unstable context, that corresponds to the liquid state. This market is characterized by the fact that coexist two distinct situations: business opportunities where competition is virtually non-existent or null and business opportunities where competition is fierce.

In thermodynamics, there are very well-defined equations and rules that allow to explain the conditions necessary for a given element to exist "to survive" in the solid state, in the gaseous state or in the liquid state.

Although this metaphor can be explored to explain the need to develop a model that allows quantifying acceptable fat levels to ensure the survival of organizations in each of the three market types, it is not proven that the level of critical organizational fat follows a similar pattern.Therefore, exploring these rules remains a challenge, as it is still not possible to find correspondences between the physical components of these equations and characteristics associated with organizational performance translated in terms of productivity and innovation capacity.

We can find in the literature several definitions for the term "lean level" of an organization. For example, Wan and Chen [20] defines the "lean level" as the performance level of the stream value compared to perfection, or according to Bayou and De Korvin [21], the "lean level" is the measure of the implementation of lean practices. For Vinodh and Cintha [22], the absence of fat is an indicator of the efficiency of the organization, since it means not only less use of the inputs to fulfil the objectives of the organization, but also an improvement in the outputs.

Thus, one of the major challenges facing this area, it is related with the development of models to assess and validate the effectiveness and efficiency of lean thinking implementation in organizations.

According to some authors, lean assessment methods can be categorized into four groups: Value Stream Mapping (VSM), Qualitative Assessment Tools, Performance Indicators and Benchmarking $[12,20]$.

According to Pakdil and Leonard [23], although there are several different methods of measuring the various perspectives of lean production, in literature there is no holistic assessment approach to determine the level of implementation of lean thinking in organizations.

As a general criticism of lean assessment methods described in the literature, we can see that each method of evaluation focuses only on a specific lean dimension and not on its entirety [21]. On the other hand, while some methods focus on the perceptions of employees, using a qualitative 
approach [24]; others use various performance metrics, creating a quantitative assessment [11, 20, 21]. However, none of the existing studies use qualitative and quantitative approaches simultaneously [23].

Currently most organizations use qualitative evaluation methods based on questionnaires or a group of metrics used simultaneously to determine the level of application/implementation of the methodologies and lean tools. The challenge of using performance indicators and metrics concerned with the assessment of the lean level of an organization, it is the ability to define a set of indicators including all dimensions of the lean approach [25]. Furthermore, the synthesis of a set of indicators in a single lean metric is also in itself a challenge due to the different measurement units [26].

Furthermore, it is worth to mention that the number of studies in literature on leanness assessment is low when compared to that in the area of lean implementation. However, the approach proposed in this paper follows the trend of lean thinking assessment literature, which is getting transformed from process-level monitoring to enterprise-level monitoring [27]. The recently published material about leanness assessment revealed that future studies in this domain would fall into two major categories, namely manufacturing leanness assessment and service leanness assessment. Thus, the framework of the proposed approach has been developed with the aim to cope with both scenarios; manufacturing and service enterprises.

\section{LEAN ASSESSMENT MODEL}

The proposed model aims to permit an assessment of lean practices, determining the lean level of an organization and to identifying the main constraints.Accordingly, the model developed utilizes the concept of fuzzy logic using triangular membership functions, to integrate both performance evaluation strands, either quantitative or qualitative in a single index, as well as to be a model able to cope with ambiguity and uncertainty of human evaluation. Thus, the model comprises the following elements:

- Model Structure - Definition of dimensions, criteria and attributes;

- Definition of linguistic variables and membership functions;

- Indicators of lean performance of an organization - Construction of fuzzy indicators to determine the lean level of an organization and to identify the main constraints.

\subsection{Model Structure}

The model architecture consists of three levels of granularity that are called, dimensions, criteria and attributes, as shown in Figure2. At the first level, it was defined three dimensions of analysis - "Customers"; "Suppliers"; and "Organization". The second level comprises thirteen criteria and in the third level are defined one hundred of attributes.

Table 1 shows the attributes defined for the criterion "5S and Visual Management". The identification of dimensions' criteria and attributes were based on the literature reviewed. Each of the elements corresponds to an indicator of lean performance, from which the lean level of the organization will be achieved. 
International Journal of Artificial Intelligence and Applications (IJAIA), Vol.8, No.5, September 2017

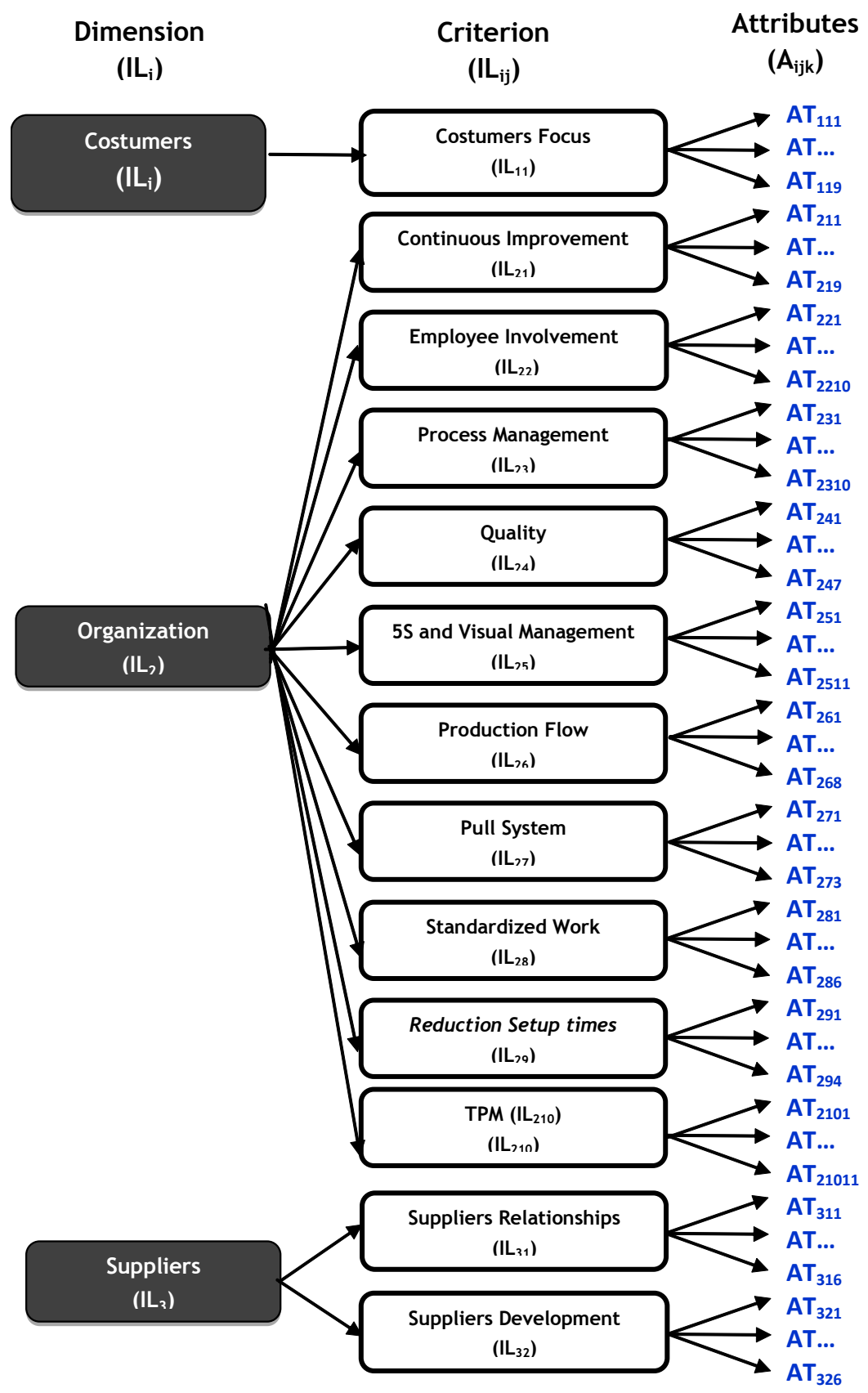

Figure 2. The model structure.

\subsection{Fuzzy Sets and Membership Functions}

Imprecision of the human systems is due to the imperfection of knowledgethat human receives (observation) from the external world. Imperfectionleads to a doubt about the value of a variable, a decision to be takenor a conclusion to be drawn for the actual system.Fuzzy logic deals with uncertainty and imprecision, and is an efficient tool for solving problems whereknowledge uncertainty may occur. Imprecise and uncertain values are usually expressed by means of linguistic terms, especially when they have been provided by or for a human being, However, the ad-hoc use of linguistic terms and the corresponding membership functions is always criticized in 
applications where fuzzy logic is used [28]. Thus, for the sake of convenience, rather than making our own definition of linguistic terms, the adoption of linguistic terms and the corresponding membership functions was chosen from the literature [28, 29, 30, 31].

In general, it is suggested that the linguistic terms to represent the behaviour of a linguistic variable do not exceed nine terms, which represent the discrimination limits of human perception [28]. Based on the literature in the field of fuzzy logic [11, 12, 13, 14] the following linguistic terms or fuzzy sets \{Excellent (E), Very Good (VG), Good (G), Satisfies (S), Satisfies Little (SL), Insufficient (I) and Weak (W) $\}$ were adopted to characterize the performance rating and it has been selected the linguistic terms \{Very High (VH), High (H), Moderately High (MH), Medium (M), Moderately Low (ML), Low (L) and Very Low (VL)\} to characterize the weighting factors, as illustrated in Table 2. The corresponding membership functions have been defined using triangular functions represented by 3 -tuples $(\mathrm{a}, \mathrm{b}, \mathrm{c})$, with parameters $\mathrm{a}<\mathrm{b}<\mathrm{c}$ and $\mathrm{b}$ representing the middle point.

Defined the fuzzy sets and the corresponding membership functions, one can now define the relative importance of each element (size, criterion and attribute), based on the strategy and policy of the organization, trend of competition, technological development, knowledge and experience of specialists [28].

Table 1. 5S Visual Management Attributes.

\begin{tabular}{|c|c|c|}
\hline Dimension & Criterion & Attributes \\
\hline Organization & $\begin{array}{l}\text { 5S and Visual } \\
\text { Management }\end{array}$ & 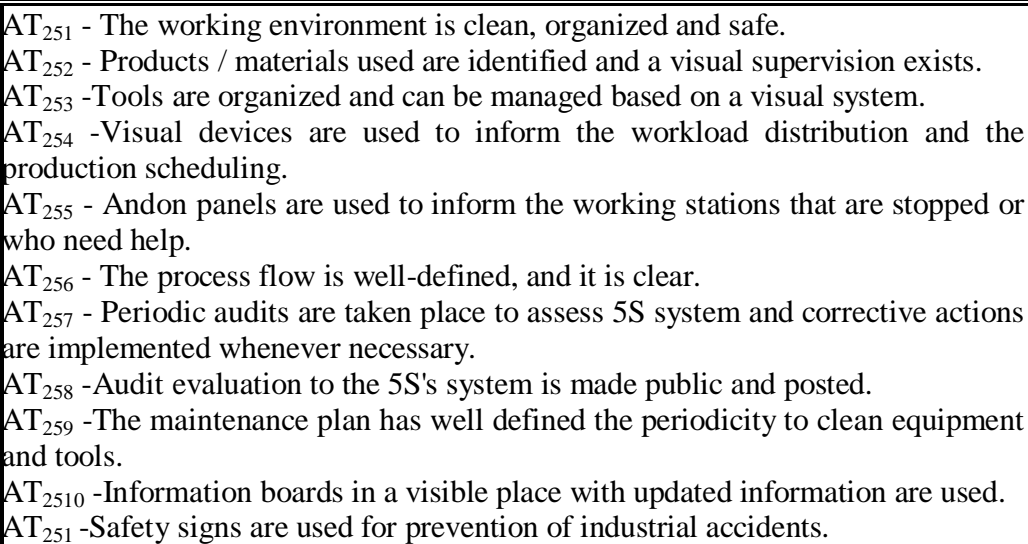 \\
\hline
\end{tabular}

Table 2. Fuzzy Sets and Membership Functions.

\begin{tabular}{cc|cc}
\hline \multicolumn{2}{c|}{ Performance Rating $(\boldsymbol{A})$} & \multicolumn{2}{c}{ Weighting Factor $(\boldsymbol{W})$} \\
\hline \hline Fuzzy Set & Membership Function & Fuzzy Set & Membership Function \\
\hline E & $(8.5 ; 9.5 ; 10)$ & VH & $(0.85 ; 0.95 ; 1)$ \\
VG & $(7 ; 8 ; 9)$ & H & $(0.7 ; 0.8 ; 0.9)$ \\
G & $(5 ; 6.5 ; 8)$ & MH & $(0.5 ; 0.65 ; 0.8)$ \\
S & $(3 ; 5 ; 7)$ & M & $(0.3 ; 0.5 ; 0.7)$ \\
SL & $(2 ; 3.5 ; 5)$ & LL & $(0.2 ; 0.35 ; 0.5)$ \\
I & $(1 ; 2 ; 3)$ & VL & $(0.1 ; 0.2 ; 0.3)$ \\
W & $(0 ; 0.5 ; 1.5)$ & & $(0 ; 0.05 ; 0.15)$ \\
\hline
\end{tabular}




\subsection{Performance Indicators of Lean Level}

This subchapter presents a detailed description of the performance metrics used to assess the lean level of an organization under the approach proposed in this paper. Thus, the following Performance Indicators (all of them being fuzzy variables) have been considered and used in the case study presented below:

- Aggregated Lean Index for each criterion $\left(L I_{i j}\right)$ - According to the definition of weighted average the Aggregated Lean Index, $L I_{i j}$, can be calculated through $W_{i j k}$ and $A_{i j k}$ variables, representing, respectively, the fuzzy weighting factors associated with each attribute (n) and the fuzzy performance ratings, by using the equation (1):

$$
L I_{i j}=\frac{\sum_{k=1}^{n}\left(W_{i j k} \times A_{i j k}\right)}{\sum_{k=1}^{n} W_{i j k}}
$$

- Aggregated Lean Index of each dimension $\left(L I_{i}\right)$ - Obtained the Aggregated Indexes for each criterion, now there is a need to integrate the values of the various criterions $(m)$ in a single index, associated with a dimension, by using the equation (2):

$$
L I_{i}=\frac{\sum_{j=1}^{m}\left(W_{i j} \times L I_{i j}\right)}{\sum_{j=1}^{m} W_{i j}}
$$

Where $W_{i j}$ is the fuzzy weighting factor associated with each criterion and $L I_{i j}$ is the aggregated fuzzy index associated also with each criterion.

- Performance Lean Index (PLI) - Obtained the $L I_{i}$, then the Performance Lean Index can be calculated using the equation (3). The PLI is a holistic fuzzy lean index consolidating in a single index the evaluations and the weighting factors. Thus, the $P L I$ represents the global lean level of an organization, where $W_{i}$ represents a fuzzy weighting factor and $L I_{i}$ stands for the aggregated lean index associated with each dimension $(l)$.

$$
P L I=\frac{\sum_{i=1}^{l}\left(W_{i} \times L I_{i}\right)}{\sum_{i=1}^{l} W_{i}}
$$

- Lean Level of an Organization $(L L O)$ - Evaluated the $P L I$, now the corresponding fuzzy value can be associated with a linguistic variable with a membership function equal or close to the membership function associated to PLI. There are several methods to associate the membership function achieved to characterize the $P L I$ with a pre-defined linguistic variable. However, it is recommended the use of the Euclidean distance method, since it is the most intuitive perception of human proximity [30]. The Euclidean distance method consists into determine the Euclidean distance between the obtained membership function and each predefined membership functions associated with each linguistic variable. Being the lean level $(L L)$ characterized by fuzzy sets with triangular membership functions, represented by 3 -tuples $(a, b, c)$, then, the Euclidean distance between the triangular membership function associated with the fuzzy set PLI and each membership function associated with the fuzzy sets predefined to characterize the fuzzy variable $L L$, can be calculated using the equation (4):

$$
\mathrm{d}\left(P L I, L_{\mathrm{i}}\right)=\sqrt{\frac{1}{3}\left[\left(\mathrm{a}_{2-} \mathrm{a}_{1}\right)^{2}+\left(\mathrm{b}_{2-} \mathrm{b}_{1}\right)^{2}+\left(\mathrm{c}_{2-} \mathrm{c}_{1}\right)^{2}\right]}
$$


International Journal of Artificial Intelligence and Applications (IJAIA), Vol.8, No.5, September 2017

With the aim to characterize the behaviour of the fuzzy variable $L L$, it was used and defined the fuzzy sets and the corresponding membership functions presented in Table 3.

Table 3. Lean Linguistic Terms - Fuzzy Sets and Membership Functions.

\begin{tabular}{lc}
\hline \multicolumn{1}{c}{ Lean linguistic terms } \\
\hline \hline \multicolumn{1}{c}{ Fets } & Membership functions \\
\hline Extremely Lean (EL) & $(7 ; 8.5 ; 10)$ \\
Very Lean (VL) & $(5.5 ; 7 ; 8.5)$ \\
Lean (L) & $(3.5 ; 5 ; 6.5)$ \\
Reasonably Lean (RL) & $(1.5 ; 3 ; 4.5)$ \\
Little Lean (LL) & $(0 ; 1.5 ; 3)$ \\
\hline
\end{tabular}

To identify the main constraints to improve the organization's lean level, it is proposed the fuzzy Importance-Performance Index (IPI) [11]. According to this index, all the attributes that are classified with a high weighting factor $\left(W_{i j k}\right)$ and then evaluated having low performance $\left(A_{i j k}\right)$, are classified as a critical constraint to improvement.

Thus, in the IPI calculation process cannot be used the values assigned to the weighting factors $W_{i j k}$, but their complementary, $\left.\left[(1,1,1)-W_{i j k}\right)\right]$, in order to not mask the results. For example, if an attribute is classified with a high $W_{i j k}$ (then, the value of $\left.\left[(1,1,1)-W_{i j k}\right]\right)$ will be low) and has a low performance evaluation $A_{i j k}$, then such an attribute is considered a critical constraint and $I P I_{i j k}$ will take a low value. For each attribute $i j k$, the fuzzy Importance-Performance Index (IPI) will be define as follows:

$$
I P I=W_{i j k}^{\prime} \times A_{i j k}
$$

where,

$$
W_{i j k}^{\prime}=(1,1,1)-W_{i j k}
$$

and, $W_{i j k}$ stands for a fuzzy weighting factor of each lean attribute.

For two triangular fuzzy numbers the subtraction, addition and multiplication operations are defined as follows:

- Fuzzy number subtraction

$$
A_{1}-A_{2}=\left(a_{1}, b_{1}, c_{1}\right)-\left(a_{2}, b_{2}, c_{2}\right)=\left(a_{1}-c_{2}, b_{1}-b_{2}, c_{1}-a_{2}\right)
$$

- Fuzzy number addition

$$
A_{1}+A_{2}=\left(a_{1}, b_{1}, c_{1}\right)+\left(a_{2}, b_{2}, c_{2}\right)=\left(a_{1}+a_{2}, b_{1}+b_{2}, c_{1}+c_{2}\right)
$$

- Fuzzy number multiplication

$$
A_{1} \times A_{2}=\left(a_{1}, b_{1}, c_{1}\right) \times\left(a_{2}, b_{2}, c_{2}\right)=\left(a_{1} \times a_{2}, b_{1} \times b_{2}, c_{1} \times c_{2}\right)
$$

Calculated the $I P I_{i j k}$ there is now the need to classify it. In this work, it was used the Chen and Hwang's left-and-right fuzzy ranking method, since it not only preserves the sort order, but also considers the absolute location of each fuzzy set [31]. In such a method to defuzify a fuzzy set, the maximum and minimum functions are given as follows:

$$
f_{\text {max }}= \begin{cases}x & 0 \leq x \leq 10 \\ 0 & \text { Otherwise }\end{cases}
$$


International Journal of Artificial Intelligence and Applications (IJAIA), Vol.8, No.5, September 2017

$$
f_{\min }=\left\{\begin{array}{cc}
1-x & 0 \leq x \leq 10 \\
0 & \text { Otherwise }
\end{array}\right.
$$

According to the above-mentioned method and considering a triangular membership function associated with the characterization of $I P I$ and defined as, $f_{I P I}: R \rightarrow[0,10]$, the left and right indexes are evaluated as follows:

$$
\begin{aligned}
& U_{L}\left(I P I_{i j k}\right)=\sup _{x}\left[f_{I P I}(x) \cap f_{\min }(x)\right] \\
& U_{R}\left(I P I_{i j k}\right)=\sup _{x}\left[f_{I P I}(x) \cap f_{\max }(x)\right]
\end{aligned}
$$

Then, the total index, which will be a crisp value being given as follows:

$$
U_{T}\left(I P I_{i j k}\right)=\frac{U_{R}+10+U_{L}}{2}
$$

\section{Case Study - Application Of The Proposed Method}

The approach proposed in this paper was applied to an organization concerned with aeronautical maintenance and manufacturing. However, before proceeding to the implementation of the proposed methodology it was carried out a training session to ensure that the evaluators were familiar with the concepts of what is meant by a lean organization to ensure adjusted results to reality. According to the literature, before proceeding to the implementation of the methodology to assess the lean level of an organization it is important to build at early stage a "consensus" with the evaluators on the object that will be subject to evaluation [29]. Defined the linguistic variables and their membership functions and assigned the relative importance of each element, follows the implementation phase of evaluation of the lean organizational performance. The fuzzy sets used to characterize the weighting factors $(w)$ have been obtained conducting a Delphy study including a set of several experts in the field of Lean, Quality, Innovation, Project Management, Management, Marketing and Logistics.

Table 4 shows an excerpt of the parameterization of the model weighting factors in order to model the previously established consensus among the evaluators.

Table 4. Weighting factors of the 5S enabler and Visual Management.

\begin{tabular}{cccccc}
\hline$L I_{i}$ & $L I_{i j}$ & $L I_{i j k}$ & $W_{i}$ & $W_{i j}$ & $W_{i j k}$ \\
\hline $\mathrm{LI}_{2}$ & $\mathrm{LI}_{25}$ & $\mathrm{LI}_{251}$ & $\mathrm{ME}$ & $\mathrm{RE}$ & $\mathrm{H}$ \\
& & $\mathrm{LI}_{252}$ & & & $\mathrm{H}$ \\
& & $\mathrm{LI}_{253}$ & & & $\mathrm{H}$ \\
& & $\mathrm{LI}_{254}$ & & & $\mathrm{H}$ \\
& & $\mathrm{LI}_{255}$ & & & $\mathrm{MH}$ \\
& & $\mathrm{LI}_{256}$ & & & $\mathrm{M}$ \\
& $\mathrm{LI}_{257}$ & & $\mathrm{ML}$ \\
& $\mathrm{LI}_{258}$ & & $\mathrm{ML}$ \\
& $\mathrm{LI}_{259}$ & & $\mathrm{MH}$ \\
\hline & $\mathrm{LI}_{2510}$ & $\mathrm{LI}_{2511}$ & & \\
\hline
\end{tabular}


Table 5 shows the result of the performance evaluation of the $5 \mathrm{~S}$ enabler and Visual Management.

Table 5. Performance evaluation of the 5S enabler and Visual Management.

\begin{tabular}{cccc}
\hline$L I_{i}$ & $L I_{i j}$ & $L I_{i j k}$ & $A_{i j k}$ \\
\hline $\mathrm{LI}_{2}$ & $\mathrm{LI}_{25}$ & $\mathrm{LI}_{251}$ & $\mathrm{~S}$ \\
& & $\mathrm{LI}_{252}$ & $\mathrm{~S}$ \\
& & $\mathrm{LI}_{253}$ & $\mathrm{~S}$ \\
& & $\mathrm{LI}_{254}$ & $\mathrm{~S}$ \\
& $\mathrm{LI}_{255}$ & $\mathrm{~S}$ \\
& $\mathrm{LI}_{256}$ & $\mathrm{G}$ \\
& $\mathrm{LI}_{257}$ & $\mathrm{I}$ \\
& $\mathrm{LI}_{258}$ & $\mathrm{I}$ \\
& $\mathrm{LI}_{259}$ & $\mathrm{G}$ \\
& $\mathrm{LI}_{2510}$ & $\mathrm{G}$ \\
& $\mathrm{LI}_{2511}$ & $\mathrm{VG}$ \\
\hline
\end{tabular}

Applying equation (1) to the values obtained in the performance evaluation of the organization the values of $L I_{i j}$ are calculated. The $L I_{i j}$ value corresponds to the aggregate index for each of the criterions. Calculated the value for the various $L I_{i j}$ and applying now the equation (2), the values for each dimension $L I_{i}$, were obtained. The $L I_{i}$ value corresponds to the aggregate index for each of the dimensions considered in the proposed approach. The obtained aggregate indices are described in Table 6 .

Table 6. Lean Performance Indicators.

\begin{tabular}{cl}
\hline \multicolumn{1}{c}{$L I_{i}$} & \multicolumn{1}{c}{$L I_{i j}$} \\
\hline Costumers $(4.14 ; 5.71 ; 7.27)$ & Costumers Focus(4.14; 5.71; 7.27) \\
Organization $(3.58 ; 5.13 ; 6.67)$ & Continuous Improvement $(3.21 ; 4.94 ; 6.65)$ \\
& Employee Involvement $(3.83 ; 5.48 ; 7.11)$ \\
& Process Management $(4.75 ; 5.71 ; 6.73)$ \\
& Quality(2.75; 4.30; 5.84) \\
& 5S and Visual Management $(3.59 ; 5.29 ; 6.93)$ \\
& Production Flow(3.86; 5.48; 6.98) \\
& Pull System(3.71; 5.54; 7.36) \\
& Standardized Work $(3.33 ; 4.98 ; 6.58)$ \\
& Setup Reduction Times $(2.93 ; 4.78 ; 6.58)$ \\
& TPM(3.49; 4.65; 5.81) \\
& Suppliers Relationship(4.18; 6.03; 7.75) \\
& Suppliers Development $(4.12 ; 5.68 ; 7.25)$ \\
\hline
\end{tabular}

Once obtained the values for the $L I_{i}$, then, the $P L I$ can be determined applying equation (3), being in the case under study $P L I=(3.88 ; 5.50 ; 7.10)$. Afterwards, as described in the last section, the determined membership function for $P L I$ should be associated with a membership function of one of the linguistic variables (fuzzy sets) pre-defined to characterize the organization's lean level and described in Table 3. Hence, using the equation (4) and as depicted in Figure3, the followingEuclidean distances between the membership function achieved for $P L I$ and each of the membership functions associated with each fuzzy set used to characterize the LLO, have been obtained: $D(P L I, E L)=3.01 ; D(P L I, V L)=1.51 ; D(P L I, L)=0.50 ; D(P L I, R L)=2.50 ; D(P L I$, $L L)=4.00$. Thus, determining the minimum value taken by $D$ can be said that the organization's lean level is "Lean", which means that the organization is in an intermediate state of lean performance. 


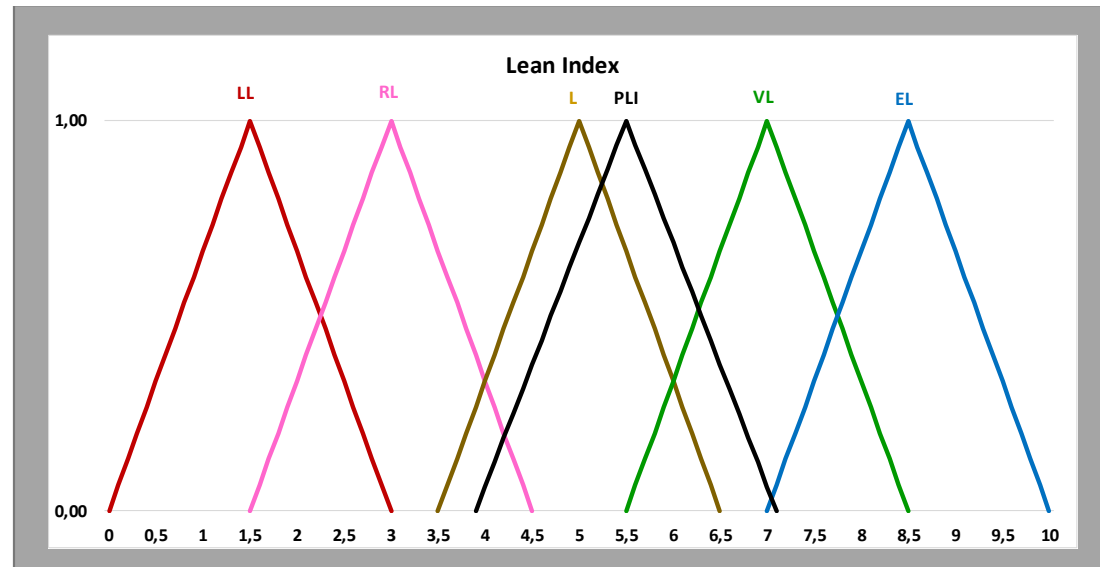

Figure 3. Membership functions of fuzzy sets used to characterize LLO and membership function associated with PLI.

To identify the main constraints to lean improvement, using equation (5) it is possible to calculate the IPI for all attributes that were evaluated. For example, the IPI for the attribute $A T_{251}$ - the work environment is clean, organized and safe, is calculated as follows:

$$
\begin{aligned}
& I P I_{251}=[(1 ; 1 ; 1)-(0.7 ; 0.8 ; 0.9)] \times(3 ; 5 ; 7) \\
& I P I_{251}=(2.1 ; 1 ; 0.3)
\end{aligned}
$$

The fuzzy value obtained for the $I P I_{251}$ should now be transformed into a crisp value. Then, through the defuzification method described in the last section, using equation (12), (13) and (14), the quoted crisp value is determined as follows:

$$
\begin{aligned}
& U_{R}\left(I P I_{251}\right)=1.89 \\
& U_{L}\left(I P I_{251}\right)=9.07 \\
& U_{T}\left(I P I_{251}\right)=\frac{1.892+10-9.065}{2}=1.41
\end{aligned}
$$

Figure 4 illustrates graphically the method used to estimate $U_{R}\left(I P I_{251}\right)$ and $U_{L}\left(I P I_{251}\right)$.

As mentioned in the Pareto principle, resources should be used to improve the critical constraints [28], and thus based on the literature, the value of 0.8 was established as the threshold to identify the critical constraints that need to be improved. Thus, since the value obtained was 1.41, corresponds to a non-critical constraint, being the critical constraints, all attributes whose value is less than or equal to 0.8 . Figure5 illustrates the 11 critical constraints identified in the organization. 
International Journal of Artificial Intelligence and Applications (IJAIA), Vol.8, No.5, September 2017

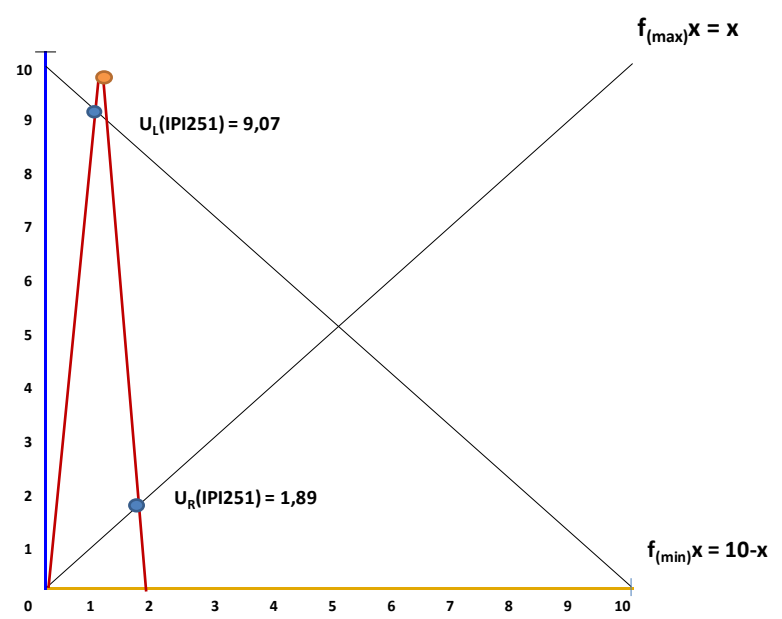

Figure 4. Graphical representation to estimate $U_{R}\left(I P I_{251}\right)$ and $U_{L}\left(I P I_{251}\right)$.

\begin{tabular}{|c|c|c|c|c|c|}
\hline$U_{T}\left(I^{\prime} I D_{i j k}\right)$ & 0,27 & 0,44 & 0,61 & 0,62 & 0,71 \\
\hline Attribute $\left(A T_{i j k}\right)$ & $\mathrm{AT}_{243}$ & $\mathrm{AT}_{217}$ & $\mathrm{AT}_{117}$ & $\mathrm{AT}_{268}$ & $\mathrm{AT}_{115}$ \\
& & $\mathrm{AT}_{227}$ & $\mathrm{AT}_{118}$ & $\mathrm{AT}_{210}$ & $\mathrm{AT}_{228}$ \\
& & & $\mathrm{AT}_{211}$ & & \\
& & & $\mathrm{AT}_{219}$ & & \\
\hline
\end{tabular}

Figure 5. Critical constraints.

The representation of the results obtained in radar graph as shown in Figure 6, can simultaneously analyse the current performance level of the various criteria. Moreover, this type of representation allows benchmarking with other organizations. Thus, the membership function associated with each fuzzy aggregate Lean Index, $L I_{i j}$, should be transformed into a crisp value by using the defuzification method mentioned in the last section, which is based on equation (12), (13) and (14). Then, the values obtained for each criterion are represented in Figure 6.

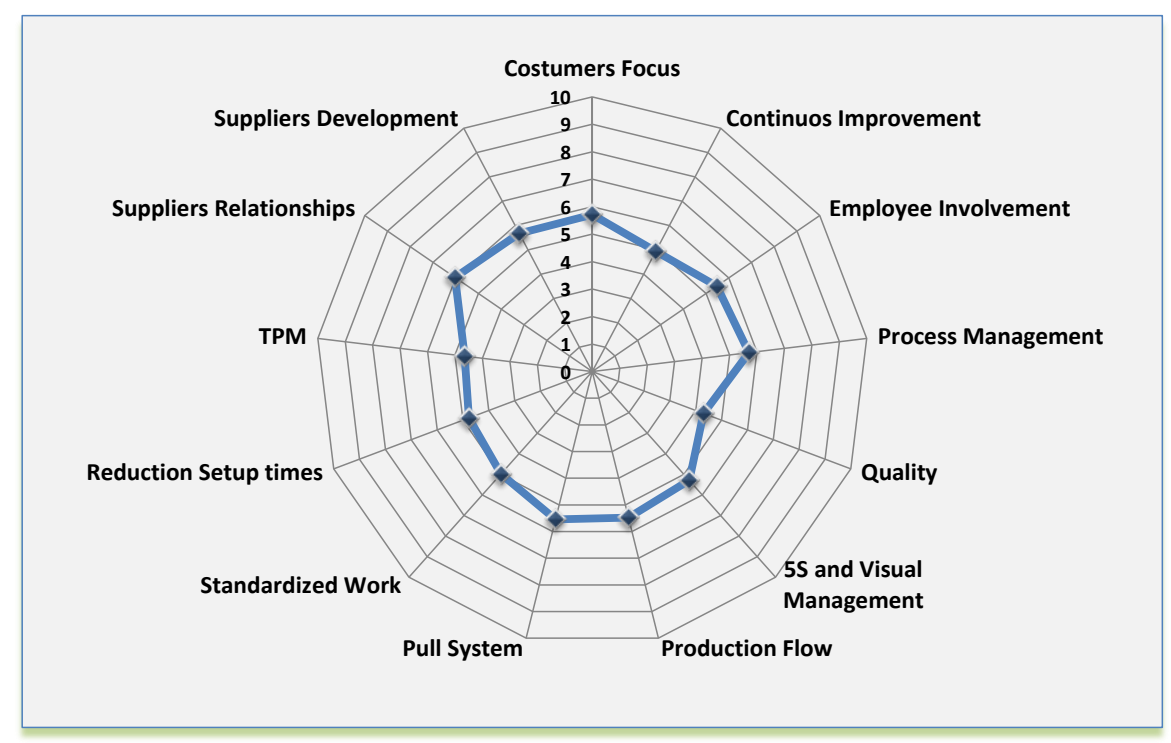

Figure 6. Fuzzy aggregate lean index for each criterion. 
The values closer to the periphery represent better performance while values closer to the centre correspond to a worse performance. For instance, considering the fuzzy aggregate Lean Index, $L I_{25}$, associated with the criterion " $5 S$ and Visual Management", the obtained corresponding value is as follows: $U_{T}\left(I L_{25}\right)=5.24$.

Aiming to obtain a graphical representation of the performance value associated with each dimension, the membership functions associated with each fuzzy aggregate lean index related to each dimension, were transformed into crisp values following the methodology previously described. In Figure 7 is represented the achieved crisp values associated with each aggregate lean index, $L I_{i}$, related to each dimension. For instance, applying the equations (9), (10) and (11) the crisp value obtained for the aggregate lean index $L I_{2}$, which corresponds to the dimension "Organization", is as follows: $U_{T}\left(L I_{25}\right)=5.11$.

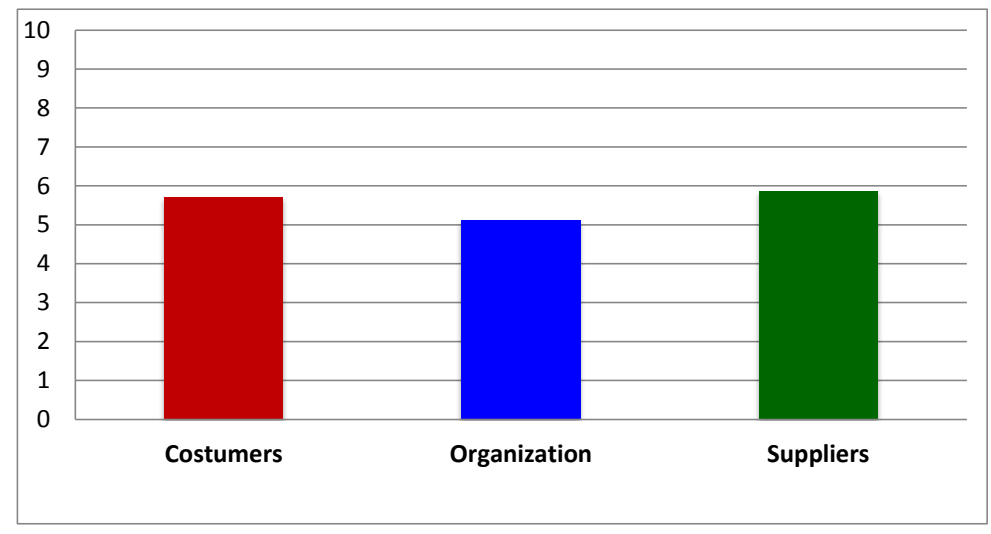

Figure 7. Aggregate lean index for each dimension.

\section{Conclusions}

Currently, markets are increasingly globalized and competitive, being constantly changing and evolving. It is in this context that companies to survive are seeking instruments to ensure their productivity gains and competitive advantages, allowing an effective and efficient management of competences based on knowledge supported by data-driven decision approaches. So many companies adopt lean thinking or lean philosophy as a survival strategy.

Organizations should make use of simultaneous approaches either of perception and measurement in order to guide their efforts to implement lean thinking more efficiently. It was in this sense that the present model was developed using a qualitative evaluation approach, including quantitative basis, supported on fuzzy logic, since it can handle the uncertainty and inaccuracy of input data, and is also applicable on the analysis of qualitative variables of a system, turning them into quantitative values. A major advantage of the developed approach, when compared with other approaches reported in the literature, it is that it can be adjusted to any type of organization regardless of its nature, size, strategy and market positioning. Moreover, the proposed model makes possible to identify systematically the constraints factors existing in the organization concerned with its lean level enhancement and, thus, provide the necessary information for the management to develop a holistic plan for continuous improvement. Furthermore, another advantage of the framework adopted to develop the organizations' lean level assessment methodology presented above, when compared with other reported approaches, it is its ability to cope with the specificities of any kind of organization, being either a manufacturing company or a services enterprise. 
The development of a framework based on fuzzy set theory was motivated in large measure by the need for a methodology able to cope with humanistic systems; that is, with systems in which human judgement behaviour and emotions play a dominant role. Viewedin this perspective, this work takes advantage of the mainfuzzy logic capabilities to perform the analysis of systems whose behaviour is based on people's skills andknowledge in management control and organizationanalysis.However, the application of fuzzy logic as a tool to support the organization assessment introduces some limitations. The membership functions of the linguistic variables (fuzzy sets) depend on the perception of the evaluator. Thus, the evaluator should be an experienced person with skills in continuous improvement or lean thinking in order to realize the importance of the model elements. The contribution of this work aims to provide a rational framework for assessing imprecise phenomena such as the case of lean assessment.

The successful results obtained with the proposed approach using a real industrial scenario, demonstrates the level of maturity of the methodology developed and allows us to envisage its application not only in research work but also in the monitoring of the implementation of lean thinking in any public or private organization.

\section{ACKNOWLEDGEMENTS}

This work was partially funded by Portuguese Funds through the Foundation for Science and Technology-FCT under the project LAETA 2015-2020, reference UID/EMS/50022/2013.

\section{REFERENCES}

[1] Gibson, R. (2011). Rethinking the future: rethinking business, principles, competition, control \& complexity, leadership, markets and the world. Nicholas Brealey Publishing.

[2] Abreu, A., andUrze, P. (2016). System thinking shaping innovation ecosystems. Open Engineering, 6(1).

[3] Camarinha-Matos, L. M., Macedo, P., and Abreu, A. (2008). Analysis of core-values alignment in collaborative networks. In Working Conference on Virtual Enterprises (pp. 53-64). Springer US.

[4] http://ec.europa.eu/research/horizon2020/index_en.cfm

[5] Koenigsaecker, G. (2012). Leading the lean enterprise transformation. CRC Press.

[6] Abreu, A., Calado, J., \& Requeijo, J. (2016). Buildings Lean Maintenance Implementation Model. Open Engineering, 6(1).

[7] Womack, J.P. ; Jones, D.T. ; Roos, D. (1990): "The machine that changed the world", Rawson Associates, New York.

[8] Mazzocato, P., Savage, C., Brommels, M., Aronsson, H., \& Thor, J. (2010). Lean thinking in healthcare: a realist review of the literature. Quality and Safety in Health Care, 19(5), 376-382.

[9] Bicheno, J. (2008). The lean toolbox for service systems. PICSIE books.

[10] Bashin, S. and Burcher, P. (2006). Lean viewed as a philosophy. Journal of Manufacturing Technology Management, Vol. 17, Issue 1, pp. 56-72.J. Clerk Maxwell, A Treatise on Electricity and Magnetism, 3rd ed., vol. 2. Oxford: Clarendon, 1892, pp.68-73.

[11] Behrouzi, F. and Wong, K.Y. (2011). Lean performance evaluation of manufacturing systems: A dynamic and innovative approach. Procedia Computer Science, Vol. 3, pp. 388-395.K. Elissa, "Title of paper if known," unpublished.

[12] Amin, M.A. (2013). A Systematic approach for selecting lean strategies and assessing leanness in manufacturing organizations'. Ph.D. Thesis, Queensland University of Technology, Australia.

[13] Bashin S. (2011): "Measuring the Leaness of an organization", International Journal of Lean Six Sigma, Vol. 2, Issue 1, pp 55-74 
[14] Behrouzi, F. ; Wong, K.Y. (2011): "Lean performance evaluation of manufacturing systems: A dynamic and innovative approach", Procedia Computer Science, Vol 3, pp 388-395.

[15] Saurin T.A. ; Marodin, G.A. ; Ribeiro, J.L.D. (2011): “A Framework for Assessing the Use of Lean Production Practices in Manufacturing Cells", International Journal of Production Research, Vol. 49, Issue 11, pp 3211-3230

[16] Womack, J. P., \& Jones, D. T. (2010). Lean thinking: banish waste and create wealth in your corporation. Simon and Schuster.

[17] Yang, M. G. M., Hong, P., and Modi, S. B. (2011). Impact of lean manufacturing and environmental management on business performance: An empirical study of manufacturing firms. International Journal of Production Economics, 129(2), 251-261.

[18] Kim, C. W. and Mauborgne, R., 2014, Blue Ocean Leadership, Harvard Business Review, 92 (5), pp. 60-72

[19] Gandellini, G. and Venanzi, D., 2011, Purple Ocean Strategy: How To Support SMEs' Recovery, Procedia Social and Behavioral Sciences, 24, pp. 1-15.

[20] Wan, H. and Chen, F. (2008). A leanness measure of manufacturing systems for quantifying impacts of lean initiatives. International Journal of Production Research, Vol. 46, Issue 23, pp. 6567-6584.

[21] Bayou, M.E. and De Korvin, A. (2008). Measuring the leanness of manufacturing systems - A case study of Ford Motor Company and General Motors. Journal of Engineering and Technology Management, Vol. 25, Issue 4, pp. 287-304.

[22] Vinodh, S. andChintha S. (2011): "Leanness assessment using multi-grade fuzzy approach", International Journal of Production Research, Vol. 49, Issue 2, pp 431-445.

[23] Pakdil, F. and Leonard, K. (2014). Criteria for a lean organisation: development of a lean assessment tool. International Journal of Production Research, Vol. 52, Issue 15, pp. 4587-4607.

[24] Fullerton, R. and Wempe, W. (2009). Lean Manufacturing, Non-financial Performance Measures, and Financial Performance. International Journal of Operations and Production Management, Vol. 29, Issue 3, pp. 214-240.

[25] Baker, P. (2008). The Role, Design and Operation of Distribution Centres in Agile Supply Chains. Ph.D. Thesis, School of Management, Cranfield University, England.

[26] Mahfouz, A. (2011). An Integrated Framework to Assess Leanness Performance in Distribution Centres. Ph.D. Thesis, Dublin Institute of Technology, England.

[27] Narayanamurthy, G. and Gurumurthy, A. (2016). Leanness assessment: a literature review. International Journal of Operations and Production Management, Vol. 36, Issue 10, pp. 1115-1160.

[28] Lin, C.-T., Chiub, H. and Tseng, Y.-H. (2006). Agility evaluation using fuzzy logic. International Journal of Production Economics, Vol. 101, Issue 2, pp. 353-368.

[29] Zanjirchi, S.M., Tooranlo, H.S. and Nejad, L.Z. (2010). Measuring Organizational Leanness Using Fuzzy Approach. Proceedings of the 2010 International Conference on Industrial Engineering and Operations Management, Dhaka, Bangladesh, pp. 144-156.

[30] Guesgen, H.W. and Albrecht, J. (2000). Imprecise reasoning in geographic information systems. Fuzzy Sets and Systems, Vol. 113, Issue 1, pp. 121-131.

[31] Chen, S.J. and Hwang, C.L. (1992). Fuzzy Multiple Attribute Decision Making Methods and Application. Lecture Notes in Economics and Mathematical Systems, Vol. 375, Springer Berlin Heidelberg.

\section{Authors}

António Abreu, before joining the academic world in 1998, he had an industrial career since 1992 in manufacturing industries with management positions.He concluded his $\mathrm{PhD}$ in 2007 in Industrial Engineering at the New University of Lisbon and he is currently professor of Industrial Engineering in the Polytechnic Institute of Lisbon (ISEL- Instituto Superior de Engenharia de Lisboa), where he now holds assistant professor position. He is member of several national and international associations, e.g. he is co- 
founder of SOCOLNET, member of the ISO/TC 258 and INSTICC . As researcher, he has been involved in several European research projects such as: VOmap, Thinkcreative and ECOLEAD. He has been involved in the organization and program committees of several national and international conferences with particular reference to PRO-VE, , MCPL, BASYS. His main research is in collaborative networked organisations, Logistics, project management, open-Innovation and lean management area.

João M. F. Calado, received his degree (5 years undergraduate course) from Instituto Superior Técnico, Technical University of Lisbon, in Electrical and Computing Engineering and the Ph.D. from The City University, London, United Kingdom, in Control Engineering, in 1986 and 1996 respectively. He joined the Maritime Machinery Department of Nautical School Infante D.Henrique, Lisbon, Portugal, in 1986, as an Assistant and was promoted to

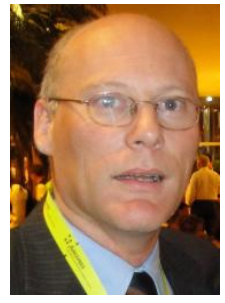
Assistant Professor, in 1991. Since 1998, he has been with the Mechanical Engineering Department of ISEL - Instituto Superior de Engenharia de Lisboa, Polytechnic Institute of Lisbon, Lisbon, Portugal, as Associate Professor being promoted to Full Professor in 2009. He is Fellow Member of the Engineers Portuguese Association, IEEE Senior Member, Member of IFAC - TC SAFEPROCESS, Member of APCA, Member of SPR and Member of Socolnet. His research and development field covers fault tolerant control, intelligent control systems, mobile robotics, rehabilitation robotics, modelling and control of manufacturing processes, multi agent systems and collaborative approaches. 
International Journal of Artificial Intelligence and Applications (IJAIA), Vol.8, No.5, September 2017

INTENTIONAL BLANK 